\title{
Second-Order Differential Equation with Multiple Delays: Oscillation Theorems and Applications
}

\author{
Shyam Sundar Santra $\mathbb{D D}^{1}$ Omar Bazighifan $\mathbb{D D}^{2,3}$ Hijaz Ahmad ${ }^{1}{ }^{4},{ }^{4}$ and Shao-Wen Yao ${ }^{5}{ }^{5}$ \\ ${ }^{1}$ Department of Mathematics, JIS College of Engineering, Kalyani 741235, India \\ ${ }^{2}$ Department of Mathematics, Faculty of Science, Hadhramout University, Hadhramout, Yemen \\ ${ }^{3}$ Department of Mathematics, Faculty of Education, Seiyun University, Hadhramout, Yemen \\ ${ }^{4}$ Department of Basic Sciences, University of Engineering and Technology, Peshawar, Pakistan \\ ${ }^{5}$ School of Mathematics and Information Science, Henan Polytechnic University, Jiaozuo 454000, China \\ Correspondence should be addressed to Shao-Wen Yao; yaoshaowen@hpu.edu.cn
}

Received 10 September 2020; Accepted 23 September 2020; Published 16 November 2020

Academic Editor: Mostafa M. A. Khater

Copyright ( 92020 Shyam Sundar Santra et al. This is an open access article distributed under the Creative Commons Attribution License, which permits unrestricted use, distribution, and reproduction in any medium, provided the original work is properly cited.

Differential equations of second order appear in physical applications such as fluid dynamics, electromagnetism, acoustic vibrations, and quantum mechanics. In this paper, necessary and sufficient conditions are established of the solutions to secondorder half-linear delay differential equations of the form $\left(\varsigma(y)\left(u^{\prime}(y)\right)^{a}\right)^{\prime}+\sum_{j=1}^{m} p_{j}(y) u^{c_{j}}\left(\vartheta_{j}(y)\right)=0$ for $y \geq y_{0}$, under the assumption $\int^{\infty}(\varsigma(\eta))^{-1 / a} \mathrm{~d} \eta=\infty$. We consider two cases when $a<c_{j}$ and $a>c_{j}$, where $a$ and $c_{j}$ are the quotient of two positive odd integers. Two examples are given to show effectiveness and applicability of the result.

\section{Introduction}

The differential equation of second order appears in models concerning biological, physical, and chemical phenomena, optimization, the mathematics of networks, and dynamical systems, see [1].

In this article, we consider the differential equation

$$
\left(\varsigma(y)\left(u^{\prime}(y)\right)^{a}\right)^{\prime}+\sum_{j=1}^{m} p_{j}(y) u^{c_{j}}\left(\vartheta_{j}(y)\right)=0, \quad \text { for } y \geq y_{0},
$$

where $a$ and $c_{j}$ are the quotient of two positive odd integers and the functions $p_{j}, \varsigma$, and $\vartheta_{j}$ are continuous that satisfy the conditions stated below:

(A1) $\vartheta_{j} \in C([0, \infty), \mathbb{R}), \vartheta_{j}(y)<y, \lim _{y \longrightarrow \infty} \vartheta_{j}(y)=\infty$

$(\mathrm{A} 2) \varsigma \in C^{1}([0, \infty), \mathbb{R}), \quad p_{j} \in C([0, \infty), \mathbb{R}) ; \quad 0<\varsigma(y)$, $0 \leq p_{j}(y)$ for all $y \geq 0$ and $j=1,2, \ldots, m ; \sum p_{j}(y)$ is not identically zero in any interval $[b, \infty)$

(A3) $\Upsilon(y)=\int_{y_{1}}^{y} \varsigma^{-1 / a}(\eta) \mathrm{d} \eta$ with $\lim _{y \longrightarrow \infty} \mathrm{Y}(y)=\infty$
(A4) The existence of a differentiable function $\vartheta_{0}$ such that

$$
\begin{aligned}
& 0<\vartheta_{0}(y)=\min \left\{\vartheta_{j}(y)\right\}, \vartheta_{0}^{\prime}(y) \geq \vartheta_{0}>0 \text { for } y \geq y_{0}, \\
& j=1,2, \ldots, m
\end{aligned}
$$

In $[2,3]$, Baculikovă and Džurina have considered

$$
\begin{gathered}
\left(\varsigma(y)\left(z^{\prime}(y)\right)^{a}\right)^{\prime}+p(y) u^{c}(\vartheta(y))=0, \\
z(y)=u(y)+q(y) u(\tau(y)), \quad y \geq y_{0},
\end{gathered}
$$

and obtained oscillation criteria for the solutions of (2) using comparison techniques when $a=c=1,0 \leq q(y)<\infty$ and $\lim _{y \rightarrow \infty} \mathrm{Y}(y)=\infty$. In the same technique, Baculikova and Džurina [4] have studied the oscillatory behavior of the solutions of (2) under the assumptions $0 \leq q(y)<\infty$ and $\lim _{y \rightarrow \infty} \Upsilon(y)=\infty$. In [?], Tripathy et al. have studied oscillatory and asymptotic behavior of (4) when $\lim _{y \rightarrow \infty} \Upsilon(y)=\infty$ and $\lim _{y \rightarrow \infty} Y(y)<\infty$ for different ranges of the neutral coefficient $q$. In [5], Bohner et al. have studied the oscillatory behavior of solutions of (2) under $a=c, \lim _{y \rightarrow \infty} \mathrm{Y}(y)<\infty$ and $0 \leq q(y)<1$. Grace et al. [6] 
have studied the oscillatory behavior of (4) when $a=c$ and $\lim _{y \rightarrow \infty} \mathrm{Y}(y)<\infty, \lim _{y \rightarrow \infty} \mathrm{Y}(y)=\infty$ and $0 \leq q(y)<1$. In [7], Li et al. have studied the oscillatory behavior of the solutions of (2), under the assumptions $\lim _{y \rightarrow \infty} \mathrm{Y}(y)<\infty$ and $q(y) \geq 0$. Karpuz and Santra [8] have studied the oscillatory behavior of

$$
\left(\varsigma(y)(u(y) q(y) u(\tau(y)))^{\prime}\right)^{\prime}+p(y) f(u(\vartheta(y)))=0,
$$

by considering the assumptions $\lim _{y \rightarrow \infty} \mathrm{Y}(y)<\infty$ and $\lim _{y \longrightarrow \infty} \mathrm{Y}(y)<\infty$ for different ranges of $q$.

For further work on the oscillation of this type of equations, we refer the readers to $[6,9-32]$. Note that the majority of works consider only sufficient conditions, and merely a few consider both necessary and sufficient conditions. Hence, the objective of this work is to establish both necessary and sufficient conditions for the oscillation of solutions of (1) without using the comparison and the Riccati techniques. In this paper, we restrict our attention to study (1), which includes the class of functional differential equations of the delay type.

Remark 1. When the domain is not specified explicitly, all functional inequalities considered in this paper are assumed to hold eventually, i.e., they are satisfied for all $y$ large enough.

\section{Necessary and Sufficient Conditions}

Lemma 1. Let (A1)-(A3) hold and that $u$ is an eventually positive solution of (1). Then, there exist $y_{1} \geq y_{0}$ and $d>0$ such that

$$
\begin{gathered}
0<u(y) \leq d \Upsilon(y), \\
\Upsilon(y)\left[\int_{y}^{\infty} \sum_{j=1}^{m} p_{j}(\zeta) u^{c_{j}}\left(\vartheta_{j}(\zeta)\right) d \zeta\right]^{1 / a} \leq u(y),
\end{gathered}
$$

for $y \geq y_{1}$.

Proof. Let $u$ be eventually positive solution of (1). Then, by (A1), there exists a $y^{*}$ such that $u(y)>0$ and $u\left(\vartheta_{j}(y)\right)>0$ for all $y \geq y^{*}$ and $j=1,2, \ldots, m$. From (1), it follows that

$$
\left(\varsigma(y)\left(u^{\prime}(y)\right)^{a}\right)^{\prime}=-\sum_{j=1}^{m} p_{j}(y) u^{c_{j}}\left(\vartheta_{j}(y)\right) \leq 0 .
$$

Therefore, $\varsigma(y)\left(u^{\prime}(y)\right)^{a}$ is nonincreasing for $y \geq y^{*}$. Next, we show the $\varsigma(y)\left(u^{\prime}(y)\right)^{a}$ is positive. By contradiction, assume that $\varsigma(y)\left(u^{\prime}(y)\right)^{a} \leq 0$ at a certain time $y \geq y^{*}$. Using that $\sum p_{j}$ is not identically zero on any interval $[b, \infty)$ and by (6), there exist $y_{1} \geq y^{*}$ such that

$$
\varsigma(y)\left(u^{\prime}(y)\right)^{a} \leq \varsigma\left(y_{1}\right)\left(u^{\prime}\left(y_{1}\right)\right)^{a}<0, \quad \text { for all } y \geq y_{1} .
$$
Then,

Recall that $a$ is the quotient of two positive odd integers.

$$
u^{\prime}(y) \leq\left(\frac{\varsigma\left(y_{1}\right)}{\varsigma(y)}\right)^{1 / a} u^{\prime}\left(y_{1}\right), \quad \text { for } y \geq y_{1}
$$

Integrating from $y_{1}$ to $y$, we have

$$
u(y) \leq u\left(y_{1}\right)+\left(\varsigma\left(y_{1}\right)\right)^{1 / a} u^{\prime}\left(y_{1}\right) \mathrm{Y}(y) .
$$

By (A3), the right-hand side approaches $-\infty$; then, $\lim _{t \rightarrow \infty} u(y)=-\infty$. This is a contradiction to the fact that $u(y)>0$. Therefore, $\varsigma(y)\left(u^{\prime}(y)\right)^{a}>0$ for all $y \geq y^{*}$. From $\varsigma(y)\left(u^{\prime}(y)\right)^{a}$ being nonincreasing, we have

$$
u^{\prime}(y) \leq\left(\frac{\varsigma\left(y_{1}\right)}{\varsigma(y)}\right)^{1 / a} u^{\prime}\left(y_{1}\right), \quad \text { for } y \geq y_{1},
$$

integrating this inequality from $y_{1}$ to $y$, and using that $u$ is continuous,

$$
u(y) \leq u\left(y_{1}\right)+\left(\varsigma\left(y_{1}\right)\right)^{1 / a} u^{\prime}\left(y_{1}\right) \mathrm{Y}(y) .
$$

Since $\lim _{y \rightarrow \infty} \mathrm{Y}(y)=\infty$, there exists a positive constant $d$ such that (4) holds.

Since $\varsigma(y)\left(u^{\prime}(y)\right)^{a}$ is positive and nonincreasing, $\lim _{y \rightarrow \infty} \varsigma(y)\left(u^{\prime}(y)\right)^{a}$ exists and is nonnegative. Integrating (1) from $y$ to $b$, we have

$$
\varsigma(b)\left(u^{\prime}(b)\right)^{a}-\varsigma(y)\left(u^{\prime}(y)\right)^{a}+\int_{y}^{b} \sum_{j=1}^{m} p_{j}(\eta) u^{c_{j}}\left(\vartheta_{j}(\eta)\right) \mathrm{d} \eta=0 .
$$

Letting limit as $b \longrightarrow \infty$, we obtain

$$
\varsigma(y)\left(u^{\prime}(y)\right)^{a} \geq \int_{y}^{\infty} \sum_{j=1}^{m} p_{j}(\eta) u^{c_{j}}\left(\vartheta_{j}(\eta)\right) \mathrm{d} \eta .
$$

Then,

$$
u^{\prime}(y) \geq\left[\frac{1}{\varsigma(y)} \int_{y}^{\infty} \sum_{j=1}^{m} p_{j}(\eta) u^{c_{j}}\left(\vartheta_{j}(\eta)\right) \mathrm{d} \eta\right]^{1 / a} .
$$

Since $u\left(y_{1}\right)>0$, integrating the above inequality yields

$$
u(y) \geq \int_{y_{1}}^{y}\left[\frac{1}{\zeta(\eta)} \int_{\eta}^{\infty} \sum_{j=1}^{m} p_{j}(\zeta) u^{c_{j}}\left(\vartheta_{j}(\zeta)\right) \mathrm{d} \zeta\right]^{1 / a} \mathrm{~d} \eta .
$$

Since the integrand is positive, we can increase the lower limit of integration from $\eta$ to $y$ and then use the definition of $\Upsilon(y)$ to obtain

$$
u(y) \geq \Upsilon(y)\left[\int_{y}^{\infty} \sum_{j=1}^{m} p_{j}(\zeta) u^{c_{j}}\left(\vartheta_{j}(\zeta)\right) \mathrm{d} \zeta\right]^{1 / a},
$$

which yields (5).

Theorem 1. Assume that there exists a constant $b_{1}$ and the quotient of two positive odd integers, such that $0<c_{j}<b_{1}<a$. If (A1)-(A3) hold, then each solution of (1) is oscillatory if and only if

$$
\int_{0}^{\infty} \sum_{j=1}^{m} p_{j}(\zeta) \mathrm{Y}^{c_{j}}\left(\vartheta_{j}(\zeta)\right) \mathrm{d} \zeta=\infty
$$


Proof. On the contrary, we assume that a solution $u$ is eventually positive. So, Lemma 1 holds, and then there exists $y_{1} \geq y_{0}$ such that

$$
u(y) \geq \mathrm{Y}(y) w^{1 / a}(y) \geq 0, \quad \text { for } y \geq y_{1},
$$

where

$$
w(y)=\int_{y}^{\infty} \sum_{j=1}^{m} p_{j}(\zeta) u^{c_{j}}\left(\vartheta_{j}(\zeta)\right) \mathrm{d} \zeta .
$$

Computing the derivative of $w$, we have

$$
w^{\prime}(y)=-\sum_{j=1}^{m} p_{j}(y) u^{c_{j}}\left(\vartheta_{j}(y)\right) .
$$

Thus, $w$ is nonnegative and nonincreasing. Since $u>0$, by (A2), it follows that $\sum_{j=1}^{m} p_{j}(y) u^{c_{j}}\left(\vartheta_{j}(y)\right)$ cannot be identically zero in any interval $[b, \infty)$; thus, $w^{\prime}$ cannot be identically zero, and $w$ cannot be constant on any interval $[b, \infty)$. Therefore, $w(y)>0$ for $y \geq y_{1}$. Computing the derivative,

$$
\left(w^{1-b_{1} / a}(y)\right)^{\prime}=\left(1-\frac{b_{1}}{a}\right) w^{-b_{1} / a}(y) w^{\prime}(y) .
$$
have

Integrating (21) from $y_{2}$ to $y$ and using that $w>0$, we

$$
\begin{aligned}
& w^{1-b_{1} / a}\left(y_{2}\right) \geq\left(1-\frac{b_{1}}{a}\right)\left[-\int_{y_{2}}^{y} w^{-b_{1} / a}(\zeta) w^{\prime}(\zeta) \mathrm{d} \zeta\right] \\
&=\left(1-\frac{b_{1}}{a}\right)\left[\int_{y_{2}}^{y} w^{-b_{1} / a}(\zeta)\left(\sum_{j=1}^{m} p_{j}(\zeta) u^{c_{j}}\left(\vartheta_{j}(\zeta)\right)\right) \mathrm{d} \zeta\right]
\end{aligned}
$$

Next, we find a lower bound for the right-hand side of (22), independent of the solution $u$. By (4) and (19), we have

$$
\begin{aligned}
u^{c_{j}}(y) & =u^{c_{j}-b_{1}}(y) u^{b_{1}}(y) \geq(d \mathrm{Y}(y))^{c_{j}-b_{1}} u^{b_{1}}(y) \\
& \geq(d \mathrm{Y}(y))^{c_{j}-b_{1}}\left(\mathrm{Y}(y) w^{1 / a}(y)\right)^{b_{1}} \\
& =d^{c_{j}-b_{1}} \mathrm{Y}^{c_{j}}(y) w^{b_{1} / a}(y), \quad \text { for } y \geq y_{2} .
\end{aligned}
$$

Since $w$ is nonincreasing, $b_{1} / a>0$ and $\vartheta_{j}(\eta)<\eta$, and it follows that

$$
\begin{aligned}
u^{c_{j}}\left(\mathcal{\vartheta}_{j}(\eta)\right) & \geq d^{c_{j}-b_{1}} \Upsilon^{c_{j}}\left(\vartheta_{j}(\eta)\right) w^{b_{1} / a}\left(\vartheta_{j}(\eta)\right) \\
& \geq d^{c_{j}-b_{1}} Y^{c_{j}}\left(\vartheta_{j}(\eta)\right) w^{b_{1} / a}(\eta) .
\end{aligned}
$$

Going back to (22), we have

$$
w^{1-b_{1} / a}\left(y_{2}\right) \geq\left(1-\frac{b_{1}}{a}\right) d^{c_{j}-b_{1}}\left[\int_{y_{2}}^{y} \sum_{j=1}^{m} p_{j}(\eta) \mathrm{Y}^{c_{j}}\left(\vartheta_{j}(\eta)\right) \mathrm{d} \eta\right] \text {. }
$$

Since $\left(1-b_{1} / a\right)>0$, by (17), the right-hand side approaches $+\infty$ as $y \longrightarrow \infty$. This contradicts (25) and completes the proof of sufficiency for eventually positive solutions.

The eventually negative solution can be dealt with similarly by introducing the variables $v=-u$.

Next, we show the necessity part by a contrapositive argument. If (17) does not hold, then, for each $\kappa>0$, there exists $y_{1} \geq y_{0}$ such that

$$
\int_{\eta}^{\infty} \sum_{j=1}^{m} p_{j}(\zeta) \mathrm{Y}^{c_{j}}\left(\vartheta_{j}(\zeta)\right) d \zeta \leq \frac{\kappa^{\left(1-c_{j} / a\right)}}{2}
$$

for all $\eta \geq y_{1}$. We define the set of continuous functions:

$$
S=\left\{u \in C([0, \infty)):\left(\frac{\kappa}{2}\right)^{1 / a} \mathrm{Y}(y) \leq u(y) \leq \kappa^{1 / a} \mathrm{Y}(y), \quad y \geq y_{1}\right\} .
$$

We define an operator $\Omega$ on $S$ by

$$
(\Omega u)(y)= \begin{cases}0, & \text { if } y \leq y_{1}, \\ \int_{y_{1}}^{y}\left[\frac{1}{\varsigma(\eta)}\left[\frac{\kappa}{2}+\int_{\eta}^{\infty} \sum_{j=1}^{m} p_{j}(\zeta) u^{c_{j}}\left(\vartheta_{j}(\zeta)\right) \mathrm{d} \zeta\right]\right]^{1 / a} \mathrm{~d} \eta, & \text { if } y>y_{1} .\end{cases}
$$

Note that when $u$ is continuous, $\Omega u$ is also continuous on $[0, \infty)$. If $u$ is a fixed point of $\Omega$, i.e., $\Omega u=u$, then $u$ is a solution of (1).

First, we estimate $(\Omega u)(y)$ from below. By (A3), we have

$$
(\Omega u)(y) \geq \int_{y_{1}}^{y}\left[\frac{1}{\varsigma(\eta)}\left[\frac{\kappa}{2}+0\right]\right]^{1 / a} \mathrm{~d} \eta=\left(\frac{\kappa}{2}\right)^{1 / a} \mathrm{Y}(y) .
$$

Now, we estimate $(\Omega u)(y)$ from above. For $u$ in $S$, we have $u^{c_{j}}\left(\vartheta_{j}(\zeta)\right) \leq\left(\kappa^{1 / a} Y\left(\vartheta_{j}(\zeta)\right)\right)^{c_{j}}$. Then, by $(26)$,

$$
\begin{aligned}
(\Omega u)(y) & \leq \int_{y_{1}}^{y}\left[\frac{1}{\varsigma(\eta)}\left[\frac{\kappa}{2}+\int_{\eta}^{\infty} \sum_{j=1}^{m} p_{j}(\zeta) u^{c_{j}}\left(\vartheta_{j}(\zeta)\right) \mathrm{d} \zeta\right]\right]^{1 / a} \mathrm{~d} \eta \\
& \leq \kappa^{1 / a} \mathrm{Y}(y) .
\end{aligned}
$$

Therefore, $\Omega$ maps $S$ to $S$.

Next, we find a fixed point for $\Omega$ in $S$. Let us define a sequence of functions in $S$ by the recurrence relation: 


$$
\begin{aligned}
v_{0}(y) & =0, \quad \text { for } y \geq y_{0}, \\
v_{1}(y) & =\left(\Omega v_{0}\right)(y)= \begin{cases}0, & \text { if } y<y_{1}, \\
\kappa^{1 / a} Y(y), & \text { if } y \geq y_{1},\end{cases} \\
v_{n+1}(y) & =\left(\Omega v_{n}\right)(y), \quad \text { for } n \geq 1, y \geq y_{1} .
\end{aligned}
$$

Note that, for each fixed $y$, we have $v_{1}(y) \geq v_{0}(y)$. Using mathematical induction, we can show that $v_{n+1}(y) \geq v_{n}(y)$. Therefore, the sequence $\left\{v_{n}\right\}$ converges pointwise to a function $v$. Using the Lebesgue Dominated Convergence Theorem, we can show that $v$ is a fixed point of $\Omega$ in $S$. This shows under assumption (26) that there is a nonoscillatory solution that does not converge to zero. This completes the proof.

Theorem 2. Assume that there exists a constant $b_{2}$ and the quotient of two positive odd integers such that $0<a<b_{2}<c_{j}$. If (A1)-(A4) hold and $\varsigma(y)$ is nondecreasing, then each solution of (1) is oscillatory if and only if

$$
\int_{y_{1}}^{\infty}\left[\frac{1}{\varsigma(\eta)} \int_{\eta}^{\infty} \sum_{j=1}^{m} p_{j}(\zeta) \mathrm{d} \zeta\right]^{1 / a} \mathrm{~d} \eta=\infty
$$

Proof. On the contrary, we assume that $u$ is an eventually positive solution that does not converge to zero. Using the same argument as in Lemma 1 , there exists $y_{1} \geq y_{0}$ such that $u\left(\vartheta_{j}(y)\right)>0$ and $\varsigma(y)\left(u^{\prime}(y)\right)^{a}$ is positive and nonincreasing. Since $\varsigma(y)>0$, so $u(y)$ is increasing for $y \geq y_{1}$. Using $u(y) \geq u\left(y_{1}\right)$, we have

$$
u^{c_{j}}(y) \geq u^{c_{j}-b_{2}}(y) u^{b_{2}}(y) \geq u^{c_{j}-b_{2}}\left(y_{1}\right) u^{b_{2}}(y),
$$

and hence

$$
u^{c_{j}}\left(\vartheta_{j}(y)\right) \geq u^{c_{j}-b_{2}}\left(y_{1}\right) u^{b_{2}}\left(\vartheta_{j}(y)\right), \quad \text { for } y \geq y_{2} .
$$

Using (34) and $\vartheta_{j}(y) \geq \vartheta_{0}(y)$, from (13), we have

$$
\varsigma(y)\left(u^{\prime}(y)\right)^{a} \geq u^{c_{j}-b_{2}}\left(y_{1}\right) u^{b_{2}}\left(\vartheta_{0}(y)\right) \int_{y}^{\infty} \sum_{j=1}^{m} p_{j}(\eta) \mathrm{d} \eta,
$$

for $y \geq y_{2}$. From $\varsigma(y)\left(u^{\prime}(y)\right)^{a}$ being nonincreasing and $\vartheta_{0}(y) \leq y$, we have

$$
\varsigma\left(\vartheta_{0}(y)\right)\left(u^{\prime}\left(\vartheta_{0}(y)\right)\right)^{a} \geq \varsigma(y)\left(u^{\prime}(y)\right)^{a} .
$$

We use this in the left-hand side of (35). Then, dividing by $\varsigma\left(\vartheta_{0}(y)\right) u^{b_{2}}\left(\vartheta_{0}(y)\right)>0$, raising both sides to the $1 / a$ power, we have

$$
\frac{u^{\prime}\left(\vartheta_{0}((y))\right)}{u^{b_{2} / a}\left(\vartheta_{0}(y)\right)} \geq\left[\frac{u^{c_{j}-b_{2}}\left(y_{1}\right)}{\varsigma\left(\vartheta_{0}(y)\right)} \int_{y}^{\infty} \sum_{j=1}^{m} p_{j}(\eta) \mathrm{d} \eta\right]^{1 / a},
$$

for $y \geq y_{2}$. Multiplying the left-hand side by $\vartheta_{0}^{\prime}(y) / \vartheta_{0} \geq 1$ and integrating from $y_{2}$ to $y$,

$$
\begin{aligned}
& \frac{1}{\vartheta_{0}} \int_{y_{2}}^{y} \frac{u^{\prime}\left(\vartheta_{0}(\eta)\right) \vartheta_{0}^{\prime}(\eta)}{u^{b_{2} / a}\left(\vartheta_{0}(\eta)\right)} \mathrm{d} \eta \geq u^{c_{j}-b_{2}}\left(y_{1}\right) \\
& \int_{y_{2}}^{y}\left[\frac{1}{\varsigma\left(\vartheta_{0}(\eta)\right)} \int_{\eta}^{\infty} \sum_{j=1}^{m} p_{j}(\zeta) \mathrm{d} \zeta\right]^{1 / a} \mathrm{~d} \eta .
\end{aligned}
$$

On the left-hand side, since $a<b_{2}$, integrating, we have

$$
\begin{aligned}
& \frac{1}{\alpha\left(1-b_{2} / a\right)}\left[z^{1-b_{2} / a}\left(\vartheta_{0}(\eta)\right)\right]_{s=y_{2}}^{y} \\
& \leq \frac{1}{\alpha\left(b_{2} / a-1\right)} z^{1-b_{2} / a}\left(\vartheta_{0}\left(y_{2}\right)\right)<\infty .
\end{aligned}
$$

On the right-hand side of (38), we use that $\varsigma\left(\vartheta_{0}(\eta)\right) \leq \varsigma(\eta)$, to conclude that (32) implies the right-hand side approaching $+\infty$, as $y \longrightarrow \infty$, which is a contradiction. Hence, the solution $u$ cannot be eventually positive.

For eventually negative solutions, we use the same change of variables as in Theorem 1 and proceed as above.

To prove the necessity part, we assume that (32) does not hold and obtain an eventually positive solution that does not converge to zero. If (32) does not hold, then for each $\kappa>0$ there exists $y_{1} \geq y_{0}$ such that

$$
\int_{y_{1}}^{\infty}\left[\frac{1}{\zeta(\eta)} \int_{\eta}^{\infty} \sum_{j=1}^{m} p_{j}(\zeta) \mathrm{d} \zeta\right]^{1 / a} \mathrm{~d} \eta<\frac{\kappa^{\left(1-c_{j} / a\right)}}{2}, \quad \forall y \geq y_{1} .
$$

We define the set of continuous function

$$
S=\left\{u \in C([0, \infty)): \frac{\kappa}{2} \leq u(y) \leq \kappa, \quad \text { for } y \geq y_{1}\right\} .
$$

Then, we define the operator:

$$
(\Omega u)(y)= \begin{cases}0, & \text { if } y \leq y_{1}, \\ \frac{\kappa}{2}+\int_{y_{1}}^{y} \frac{1}{\zeta(\eta)}\left[\int_{\eta}^{\infty} \sum_{j=1}^{m} p_{j}(\zeta) u^{c_{j}}\left(\vartheta_{j}(\zeta)\right) \mathrm{d} \zeta\right]^{1 / a} \mathrm{~d} \eta, & \text { if } y>y_{1} .\end{cases}
$$

Note that if $u$ is continuous, $\Omega u$ is also continuous at $y=y_{1}$. Also note that if $\Omega u=u$, then $u$ is solution of (1).
First, we estimate $(\Omega u)(y)$ from below. Let $u \in M$, and we have $(\Omega u)(y) \geq \kappa / 2+0$, on $\left[y_{1}, \infty\right)$. 
Now, we estimate $(\Omega u)(y)$ from above. Let $u \in M$. Then, $u \leq \kappa$, and by (40), we have

$$
(\Omega u)(y) \leq \frac{\kappa}{2}+\kappa^{c_{j} / a} \int_{y_{1}}^{y}\left[\frac{1}{\varsigma(\eta)} \int_{\eta}^{\infty} \sum_{j=1}^{m} p_{j}(\zeta) \mathrm{d} \zeta\right]^{1 / a} \mathrm{~d} \eta \leq \frac{\kappa}{2}+\frac{\kappa}{2}=\kappa .
$$

Therefore, $\Omega$ maps $S$ to $S$. To find a fixed point for $\Omega$ in $S$, we define a sequence of functions by the recurrence relation:

$$
\begin{aligned}
v_{0}(y) & =0, \quad \text { for } y \geq y_{0}, \\
v_{1}(y) & =\left(\Omega v_{0}\right)(y)=1, \quad \text { for } y \geq y_{1}, \\
v_{n+1}(y) & =\left(\Omega v_{n}\right)(y), \quad \text { for } n \geq 1, y \geq y_{1} .
\end{aligned}
$$

Note that, for each fixed $y$, we have $v_{1}(y) \geq v_{0}(y)$. Using mathematical induction, we can prove that $v_{n+1}(y) \geq v_{n}(y)$. Therefore, $\left\{v_{n}\right\}$ converges pointwise to a function $v$ in $S$. Then, $v$ is a fixed point of $\Omega$ and a positive solution of (1). The proof is completed.

Example 1. Consider

$$
\left(e^{-y}\left(u^{\prime}(y)\right)^{11 / 3}\right)^{\prime}+\frac{1}{y+1}(u(y-2))^{1 / 3}+\frac{1}{y+2}(u(y-1))^{5 / 3}=0 .
$$

Here, $\quad a=11 / 3, \quad \varsigma(y)=e^{-y}, \quad \vartheta_{1}(y)=y-2$, $\vartheta_{2}(y)=y-1, \Upsilon(y)=\int_{y_{1}}^{y} e^{11 \eta / 3} \mathrm{~d} \eta=(3 / 11)\left(e^{11 y / 3}-e^{11 y_{1} / 3}\right)$, $c_{1}=1 / 3$, and $c_{2}=5 / 3$. For $b=7 / 3$, we have $0<\max \left\{c_{1}\right.$, $\left.c_{2}\right\}<b<a$. To check (17), we have

$$
\begin{aligned}
& \int_{0}^{\infty} \sum_{j=1}^{m} p_{j}(s) \Upsilon^{c_{j}}\left(\vartheta_{j}(s)\right) \mathrm{d} s \geq \int_{0}^{\infty} p_{1}(s) \Upsilon^{\alpha_{1}}\left(\vartheta_{1}(s)\right) \mathrm{d} s \\
& \quad=\int_{0}^{\infty} \frac{1}{\eta+1}\left(\frac{3}{11}\left(e^{11(s-2) / 3}-e^{11 y_{1} / 3}\right)\right)^{1 / 3} \mathrm{~d} s=\infty .
\end{aligned}
$$

So, every condition of Theorem 1 holds true. Therefore, all solution of (45) is oscillatory.

\section{Example 2. Consider}

$$
\left(\left(u^{\prime}(y)\right)^{1 / 3}\right)^{\prime}+y(u(y-2))^{7 / 3}+(y+1)(u(y-1))^{11 / 3}=0 .
$$

Here, $a=1 / 3, \varsigma(y)=1, \vartheta_{1}(y)=y-2, \vartheta_{2}(y)=y-1$, $c_{1}=7 / 3$, and $c_{2}=11 / 3$. For $b=5 / 3$, we have $\min \left\{c_{1}, c_{2}\right\}>b>a$. To check (32), we have

$$
\begin{gathered}
\int_{y_{0}}^{\infty}\left[\frac{1}{\varsigma(s)} \int_{s}^{\infty} \sum_{j=1}^{m} p_{j}(\zeta) \mathrm{d} \zeta\right]^{1 / a} \mathrm{~d} s \\
\geq \int_{y_{0}}^{\infty}\left[\frac{1}{\varsigma(s)} \int_{s}^{\infty} p_{1}(\zeta) \mathrm{d} \zeta\right]^{1 / a} \mathrm{~d} s \\
\geq \int_{2}^{\infty}\left[\int_{s}^{\infty} \zeta \mathrm{d} \zeta\right]^{3} \mathrm{~d} s=\infty .
\end{gathered}
$$

So, every conditions of Theorem 2 hold true. Thus, all solutions of (47) is oscillatory.

\section{Conclusion}

This work aims to study the oscillatory behavior of secondorder neutral nonlinear differential equation. The obtained oscillation theorems complement the well-known oscillation results present in the literature.

\section{Data Availability}

The data used to support the findings of the study are available from the corresponding author upon request.

\section{Conflicts of Interest}

The authors declare that they have no conflicts of interest.

\section{Acknowledgments}

This work was supported by the National Natural Science Foundation of China (no. 71601072) and Key Scientific Research Project of Higher Education Institutions in Henan Province of China (no. 20B110006).

\section{References}

[1] J. K. Hale, Theory of Functional Differential Equations, Springer-Verlag, Berlin, Germany, 1977.

[2] B. Baculíková and J. Džurina, "Oscillation theorems for second order neutral differential equations," Computers \& Mathematics with Applications, vol. 61, no. 1, pp. 94-99, 2011.

[3] J. Džurina, "Oscillation theorems for second-order advanced neutral differential equations," Tatra Mountains Mathematical Publications, vol. 48, pp. 61-71, 2011.

[4] B. Baculíková and J. Džurina, "Oscillation theorems for second-order nonlinear neutral differential equations," Computers \& Mathematics with Applications, vol. 62, no. 12, pp. 4472-4478, 2011.

[5] M. Bohner, S. Grace, and I. Jadlovská, "Oscillation criteria for second-order neutral delay differential equations," Electronic Journal of Qualitative Theory of Differential Equations, vol. 60, pp. 1-12, 2017.

[6] S. R. Grace, J. Džurina, I. Jadlovska, and T. Li, “An improved approach for studying oscillation of second-order neutral delay differential equations," Journal of Inequalities and Applications, vol. 2018, p. 11, 2018.

[7] H. Li, Y. Zhao, and Z. Han, "New oscillation criterion for Emden-Fowler type nonlinear neutral delay differential equations," Journal of Computational and Applied Mathematics, vol. 60, no. 1-2, pp. 191-200, 2019.

[8] B. Karpuz and S. S. Santra, "Oscillation theorems for secondorder nonlinear delay differential equations of neutral type," Hacettepe Journal of Mathematics and Statistics, vol. 48, no. 3, pp. 633-643, 2019.

[9] R. P. Agarwal, M. Bohner, T. Li, and C. Zhang, "Oscillation of second-order differential equations with a sublinear neutral term," Carpathian Journal of Mathematics, vol. 30, pp. 1-6, 2014.

[10] R. P. Agarwal, M. Bohner, T. Li, and C. Zhang, "Oscillation of second-order Emden-Fowler neutral delay differential 
equations," Annals of Pure and Applied Mathematics, vol. 193, no. 4, pp. 1861-1875, 2014.

[11] R. P. Agarwal, M. Bohner, T. Li, and C. Zhang, "Even-order half-linear advanced differential equations: improved criteria in oscillatory and asymptotic properties," Applied Mathematics and Computation, vol. 266, pp. 481-490, 2015.

[12] R. P. Agarwal, C. Zhang, and T. Li, "Some remarks on oscillation of second order neutral differential equations," Applied Mathematics and Computation, vol. 274, pp. 178-181, 2016.

[13] B. Baculikova, T. Li, and J. Dzurina, "Oscillation theorems for second order neutral differential equations," Electronic Journal of Qualitative Theory of Differential Equations, vol. 74, no. 74, pp. 1-13, 2011.

[14] G. E. Chatzarakis, S. R. Grace, I. Jadlovská, T. Li, and E. Tunç, "Oscillation criteria for third-order emden-fowler differential equations with unbounded neutral coefficients," Complexity, vol. 2019, pp. 1-7, Article ID 5691758, 2019.

[15] J. Džurina, S. R. Grace, I. Jadlovska, and T. Li, "Oscillation criteria for second-order Emden-Fowler delay differential equations with a sublinear neutral term," Mathematische Nachrichten, vol. 293. , 2019 In press.

[16] T. Li and Y. V. Rogovchenko, "Oscillatory behavior of secondorder nonlinear neutral differential equations," Abstract and Applied Analysis, vol. 2014, pp. 1-8, Article ID 594190, 2014.

[17] T. Li and Y. V. Rogovchenko, "Oscillation of second-order neutral differential equations," Mathematische Nachrichten, vol. 288, no. 10, pp. 1150-1162, 2015.

[18] M. A. Ragusa, "Elliptic boundary value problem in vanishing mean oscillation hypothesis," Commentationes Mathematicae Universitatis Carolinae, vol. 40, no. 4, pp. 651-663, 1999.

[19] M. A. Ragusa, "Homogeneous herz spaces and regularity results," Nonlinear Analysis: Theory, Methods and Applications, vol. 71, 2009.

[20] S. Pinelas and S. S. Santra, "Necessary and sufficient condition for oscillation of nonlinear neutral first-order differential equations with several delays," Journal of Fixed Point Theory and Applications, vol. 20, no. 27, pp. 1-13, 2018.

[21] S. Pinelas and S. S. Santra, "Necessary and sufficient conditions for oscillation of nonlinear first-order forced differential equations with several delays of neutral type," Analysis, vol. 39, no. 3, pp. 97-105, 2019.

[22] Y. Qian and R. Xu, "Some new oscillation criteria for higherorder quasi-linear neutral delay differential equations," Differential Equations \& Applications, vol. 3, no. 3, pp. 323-335, 2011.

[23] S. S. Santra, "Existence of positive solution and new oscillation criteria for nonlinear first-order neutral delay differential equations," Differential Equations \& Applications, vol. 8, no. 1, pp. 33-51, 2016.

[24] S. S. Santra, "Oscillation analysis for nonlinear neutral differential equations of second-order with several delays," Mathematica, vol. 59, no. 82, pp. 111-123, 2017.

[25] S. S. Santra, "Oscillation analysis for nonlinear neutral differential equations of second-order with several delays and forcing term," Mathematica, vol. 61, no. 84, pp. 63-78, 2019.

[26] S. S. Santra, "Necessary and sufficient condition for the solutions of first-order neutral differential equations to be oscillatory or tend to zero," Kyungpook Mathematical Journal, vol. 59, pp. 73-82, 2019.

[27] S. S. Santra, "Necessary and sufficient condition for oscillatory and asymptotic behaviour of second-order functional differential equations," Kragujevac Journal of Mathematics, vol. 44, no. 3, pp. 459-473, 2020.
[28] S. S. Santra, "Necessary and sufficient conditions for oscillatory and asymptotic behaviour of solutions to second-order nonlinear neutral differential equations with several delays," Tatra Mountains Mathematical Publications, vol. 75, no. 1, pp. 121-134, 2020.

[29] J. S. W. Wong, "Necessary and sufficient conditions for oscillation of second order neutral differential equations," Journal of Mathematical Analysis and Applications, vol. 252, no. 1, pp. 342-352, 2000.

[30] Q. Yang and Z. Xu, "Oscillation criteria for second order quasilinear neutral delay differential equations on time scales," Computers \& Mathematics with Applications, vol. 62, no. 10, pp. 3682-3691, 2011.

[31] L. Ye and Z. Xu, "Oscillation criteria for second order quasilinear neutral delay differential equations," Applied Mathematics and Computation, vol. 207, no. 2, pp. 388-396, 2009.

[32] C. Zhang, R. P. Agarwal, M. Bohner, and T. Li, "Oscillation of second-order nonlinear neutral dynamic equations with noncanonical operators," Bulletin of the Malaysian Mathematical Sciences Society, vol. 38, no. 2, pp. 761-778, 2015. 